\title{
Distributed Accelerated Proximal Coordinate Gradient Methods
}

\author{
Yong Ren, Jun Zhu* \\ Center for Bio-Inspired Computing Research \\ State Key Lab for Intell. Tech. \& Systems \\ Dept. of Comp. Sci. \& Tech., TNList Lab, Tsinghua University \\ renyong15@mails.tsinghua.edu.cn; dcszj@tsinghua.edu.cn
}

\begin{abstract}
We develop a general accelerated proximal coordinate descent algorithm in distributed settings (DisAPCG) for the optimization problem that minimizes the sum of two convex functions: the first part $f$ is smooth with a gradient oracle, and the other one $\Psi$ is separable with respect to blocks of coordinate and has a simple known structure (e.g., $L_{1}$ norm). Our algorithm gets new accelerated convergence rate in the case that $f$ is strongly convex by making use of modern parallel structures, and includes previous non-strongly case as a special case. We further present efficient implementations to avoid full-dimensional operations in each step, significantly reducing the computation cost. Experiments on the regularized empirical risk minimization problem demonstrate the effectiveness of our algorithm and match our theoretical findings.
\end{abstract}

\section{Introduction}

We consider the following optimization problem with a composite objective function:

$$
\min _{x \in \mathbb{R}^{N}} F(x):=f(x)+\Psi(x),
$$

where $f$ and $\Psi$ are proper and lower semi-continuous convex functions. We further assume that $f$ is differentiable on $\mathbb{R}^{N}$, and $\Psi$ has a simple blockwise separable structure $\Psi(x)=\sum_{i=1}^{n} \Psi_{i}\left(x_{i}\right)$, where $x_{i}$ is the $i$-th block of $x$ with cardinality $N_{i}$. This problem is ubiquitous in machine learning, where $f(x)$ denotes the loss and $\Psi$ represents some constraints or regularizations. Given a set $\mathcal{D}$ of i.i.d data, a typical loss function $f$ has the following form

$$
f(x)=\sum_{A_{j} \in \mathcal{D}} l\left(x ; A_{j}\right)
$$

where $l$ is some smoothed loss function such as the smoothed hinge loss [Lin et al., 2014]. The choice of $\Psi$ depends on the requirements of certain problems, such as bound constraints (e.g., $\Psi_{i}(x)=0$ for $x \in[0,1]$ and $\infty$ otherwise) or regularizations for a special purpose (e.g., $L_{1}$-regularizer for sparsity). $f(x)$ can be strongly convex or not. The strong convexity property usually means a faster (linear) convergence rate

\footnotetext{
${ }^{*}$ Corresponding author
}

and hence is interested in many cases (e.g., ridge regression). We aim to develop a general accelerated coordinate descent method to solve problem (1) under distributed settings, and accelerate the convergence rate by making use of the parallel structure.

\subsection{Related Work and Motivation}

Coordinate descent (CD) methods are popular optimization algorithms to handle such problems that can break down to small pieces since they can usually make use of special structures underlying the problems. At each iteration $t$, the basic CD method chooses one block of coordinate $x_{i_{t}}$ to sufficiently reduce the objective value while keeping the other blocks fixed. There are two common strategies for choosing such a block-the cyclic scheme and the randomized scheme, where the former chooses $i_{t}$ in a cyclic fashion (i.e., $i_{t+1}=i_{t} \bmod n+1$ ), while the latter chooses $i_{t}$ uniformly or via some distributions. The randomized scheme is more common since it enjoys both theoretical and practical benefits [Wright, 2015]. Due to their superior convergence property, CD algorithms have been widely used in many practical problems, especially in some regimes that need relatively high accurate solutions [Wright, 2015].

To improve the performance of the basic CD algorithm, many variants have been proposed. Among them, Nesterov's acceleration technique [Nesterov, 1983], which is proven to be an "optimal" first order (gradient) method in convergence rate, is an important line. Specially, Nesterov [2012] developed an accelerated randomized CD method for minimizing unconstrained smooth functions (i.e., with $\Psi(x)=0$ ), which is the first one that applies acceleration techniques to $\mathrm{CD}$ methods. Later one, Lu and Xiao [2013] gave an improved version with a sharper convergence analysis. For the more general problem (1), Fercoq and Richtarik [2015] proposed the APPROX algorithm for solving functions $f(x)$ without strong convexity to obtain an accelerated sublinear convergence rate and Lin [2014] proposed a general APCG algorithm to obtain an accelerated convergence rate for $f(x)$ either with strong convexity or not.

Another important line is to utilize parallel computing architectures to accelerate the $\mathrm{CD}$ algorithms. Here we focus on the synchronous case, where multiple blocks of coordinates are sampled and gradients are then computed in each iteration, running in parallel on multiple processors. After 
that, a barrier synchronization step is set to update the coordinates, see a series of recent work in parallel and distributed settings [Bradley et al., 2011; Necoara and Clipici, 2013; Richtarik and Takac, 2013a]. To meet the requirements of the big-data challenge, combining the acceleration technique with advanced parallel computing architectures seems to be natural to leverage their advantages respectively. For the general problem (1), the APPROX algorithm is parallel in nature to deal with $f(x)$ without strong convexity. For a special case of (1) called empirical risk minimization (ERM) problem, Shalev-Shwartz and Zhang [2014] proposed an innerouter loop optimization scheme to obtain an accelerated convergence rate with a strong convexity assumption, where the inner loop is easily parallelized using existing techniques [Takac and Richtarik, 2015]. However, the parallel algorithm for problem (1) in the strongly convex case is still unexplored.

In this paper, we propose a new distributed accelerated coordinate gradient descent method (DisAPCG) to solve the general form (1) for $f(x)$ with or without strong convexity. Our algorithm lies in the line of Nesterov acceleration methods, with new carefully modified Nesterov's sequences to adapt into distributed settings. Our algorithm includes APPROX as a special case when the function does not have a strong convexity assumption, where a sublinear convergence rate is obtained. In the strong convex case, we obtain an accelerated linear convergence rate, thanks to the parallel structure. Furthermore, we propose an efficient implementation to avoid full-dimensional vector operations, which reduces the updating cost in each iteration. For practical use, we apply our algorithm to the ERM problem and find a significant improvement comparing with several other distributed CD solvers.

\subsection{Outline of The Paper}

In Section 2, we introduce the notations and assumptions, present the general DisAPCG method, analyze its convergence rate with or without strong convexity, and show the gain by leveraging parallel structures. In Section 3, we present an equivalent version of the general algorithm to avoid full-dimensional manipulation in many cases. In Section 4, we apply our DisAPCG method to the widely studied ERM problem. Besides, we use experiments on a smoothed version of SVM problems to demonstrate the effectiveness of our algorithm. Finally, we conclude in Section 5.

\section{The DisAPCG Method}

\subsection{Notations, Assumptions and Settings}

For an $N$-dimensional vector $x \in \mathbb{R}^{N}$, let $\Omega=$ $\left\{x_{i} \in \mathbb{R}^{N_{i}}\right\}_{i=1}^{n}$ denote a partition of the coordinates with $\sum_{i=1}^{n} N_{i}=N$. Let $U=\left[U_{1}, \ldots, U_{n}\right]$ be an $N \times N$ permutation matrix with $U_{i} \in \mathbb{R}^{N \times N_{i}}$. Then, we have

$$
x=\sum_{i=1}^{n} U_{i} x_{i}, \quad \text { and } x_{i}=U_{i}^{\top} x, \quad i \in[n] .
$$

Without losing generality, we assume that $U$ is the identity matrix. Our distributed setting is similar as in [Richtarik and Takac, 2013a]. Suppose that the cluster consists of $K$ nodes and the blocks of coordinates (features) are uniformly distributed in each node (i.e., each node keeps and updates $n / K$ blocks of coordinates). For simplicity, we assume that $n$ is divisible by $K$. We denote $S_{k} \subset \Omega$ as the collection of blocks of coordinates that are distributed in the $k$-th node and we have $\cup_{k=1}^{K} S_{k}=\Omega$. In the meanwhile, all the data describing the features $S_{k}$ are stored in the $k$-th node.

Following [Lin et al., 2014], for any $x \in \mathbb{R}^{N}$, the partial gradient of $f$ with respect to $x_{i}$ is defined as

$$
\nabla_{i} f(x)=U_{i}^{\top} \nabla f(x), \quad i \in[n],
$$

where $[n]:=\{1, \ldots n\}$ denotes the set of integers from 1 to $n$. We make the following assumptions, which are commonly used in the literature of coordinate descent methods [Fercoq and Richtarik, 2015].

Assumption 1. The gradient of $f(x)$ is block-wise Lipschitz continuous with constants $L_{i}$ :

$\left\|\nabla_{i} f\left(x+U_{i} h_{i}\right)-\nabla_{i} f(x)\right\|_{2} \leq L_{i}\left\|h_{i}\right\|_{2}, \quad x \in \mathbb{R}^{N}, \quad h_{i} \in \mathbb{R}^{N_{i}}$.

For convenience, we denote $\|x\|_{L}=\left(\sum_{i=1}^{n} L_{i}\left\|x_{i}\right\|_{2}^{2}\right)^{1 / 2}$ as

the $L_{2}$-norm blockwisely weighted by the coefficients $L_{i}$.

Assumption 2. There exists $\mu \geq 0$ such that for all $y \in \mathbb{R}^{N}$ and $x \in \operatorname{dom}(\Psi)$ :

$$
f(y) \geq f(x)+\langle\nabla f(x), y-x\rangle+\frac{\mu}{2}\|y-x\|_{L}^{2} .
$$

An immediate consequence of Assumption 1 is $f\left(x+U_{i} h_{i}\right) \leq f(x)+\left\langle\nabla_{i} f(x), h_{i}\right\rangle+\frac{L_{i}}{2}\left\|h_{i}\right\|_{2}^{2}, \forall h_{i} \in \mathbb{R}^{N_{i}}$, which bounds the variation of $f(x)$ when a single block changes. In distributed settings, as more than one blocks vary in each iteration, we need the following lemma to bound the total variation of the function $f(x)$.

Lemma 1. ([Richtarik and Takac, 2013b]) Assume that $f$ satisfies Assumption 1. For all $x, h \in \mathbb{R}^{N}$, we have

$$
f(x+h) \leq f(x)+\langle\nabla f(x), h\rangle+\frac{|S u p p(h)|}{2}\|h\|_{L}^{2},
$$

where $\operatorname{Supp}(h):=\left\{i \in[n]: h^{(i)} \neq 0\right\}$ is the set of blocks that are not equal to zero.

The above bound is tight in general. Suppose that in distributed settings, we alter $\kappa$ blocks in each iteration and define

$$
\|\cdot\|_{L_{\kappa}}^{2}=\kappa\|\cdot\|_{L}^{2}
$$

Then, the bound gives that for $x, y \in \mathbb{R}^{N}$,

$$
f(y) \geq f(x)+\langle\nabla f(x), y-x\rangle+\frac{\mu_{\kappa}}{2}\|y-x\|_{L_{\kappa}}^{2},
$$

and Assumption 1 implies that

$$
f(x+h) \leq f(x)+\langle\nabla f(x), h\rangle+\frac{1}{2}\|h\|_{L_{\kappa}}^{2},
$$

where $\mu_{\kappa}=\mu / \kappa$.

\subsection{The DisAPCG Algorithm}

The general DisAPCG algorithm is summarized in Alg.1. We start $K$ processors and each processor $k$ runs Alg.1 simultaneously. The algorithm maintains a non-increasing step size $\alpha_{t}$ and intermediate variable $y^{(t)}$, similarly in the original Nesterov's method. At iteration $t$, each node samples multiple blocks of coordinates and computes the intermediate variable $z^{(t)}$ using a proximal gradient step, as in step 4 . Finally, 
the coordinates are updated in a synchronized manner in step 5 . We make more comments on several key steps as follows.

Step1: $S_{k}^{(t)}$ collects the indices of the coordinates to be updated for node $k$. The total number of updated coordinates in one iteration is $\kappa=\tau K$, where $\tau$ is the mini-batch size that every node samples at each iteration. Note that the sample procedure is not equivalent to uniformly sample $\kappa$ blocks of coordinates from all since they are stored locally.

Our algorithm is essentially in a mini-batch manner. When $K=1$, we can merge several small blocks into a larger one and use [Lin et al., 2014] directly (i.e., uniformly sample one large block at each iteration), and hence the mini-batch analysis is not necessary. However, such merging can not be done in the distributed settings, since every node needs to sample their own data, not as a whole.

Step2: The original Nesterov's sequence is computed as $n^{2} \alpha_{t}^{2}=\left(1-\alpha_{t}\right) \gamma_{t}+\alpha_{t} \mu$. Our new sequence considers the influence introduced by multi-block alteration in one iteration. Similar properties hold as the original ones.

Step3: The Reduce operator is similar to MPI::AllReduce, where $y^{(t)}$ is first computed by gathering coordinates from all nodes and then broadcasted to all nodes. This is a crucial step since $y^{(t)}$ is of size $O(N)$ and hence introduces the most part of communication cost. $y^{(t)}$ is used to compute the gradient, however, one can further reduce the communication cost by exploring the special structure of $f(x)$, as we shall see.

Step5: The update step involves full-dimensional operations since $z^{(t+1)}, z^{(t)}$ and $y^{(t)}$ are dense in general and the similar problem exists for the computation of $y^{(t)}$. This issue will be dealt with by proposing an efficient and equivalent algorithm in Section 3.

\subsection{Convergence Analysis}

We analyze the convergence rate of Alg.1. The main theorem is as follows. We defer the full proof into appendix.

Theorem 1. Suppose that Assumptions 1 and 2 hold. Let $F^{*}$ be the optimal value of problem (1) and $\left\{x^{(t)}\right\}$ be the sequence generated by the DisAPCG method. Then for any $t \geq 0$, the following holds:

$$
\begin{aligned}
\mathbb{E}\left[F\left(x^{(t)}\right]-F^{*} \leq \min \right. & \left\{\left(1-\frac{\sqrt{\kappa \mu}}{n}\right)^{t},\left(\frac{2 n}{2 n+t \kappa \sqrt{\gamma_{0}}}\right)^{2}\right\} \\
& \left(F\left(x^{(0)}\right)-F^{*}+\frac{\gamma_{0}}{2} \kappa R_{0}^{2}\right),
\end{aligned}
$$

where $R_{0}:=\min _{x^{*} \in X^{*}}\left\|x^{(0)}-x^{*}\right\|_{L}^{2}$, and $X^{*}$ is the set of optimal solutions of problem (1).

The two terms $\left(1-\frac{\sqrt{\kappa \mu}}{n}\right)^{t}$ and $\left(\frac{2 n}{2 n+t \kappa \sqrt{\gamma_{0}}}\right)^{2}$ correspond to the strong convex and non-strong convex cases respectively. For the non-strongly convex case, a slight change of the proof can remove the $\kappa$ in the last term of (7) and then we recover the convergence rate in [Fercoq and Richtarik, 2015] as a special case.

For the strongly convex case, we get an accelerated linear convergence rate, as the following corollary shows:
Algorithm 1 The DisAPCG algorithm.

Input: $x^{(0)} \in \operatorname{dom}(\Psi)$ and convexity parameter $\mu_{\kappa} \geq 0$.

Initialize: set $z^{(0)}=x^{(0)}$ and choose $0<\gamma_{0} \in\left[\mu_{\kappa}, 1\right]$.

Start $K$ nodes with their corresponding data and coordinates. for $t=0,1,2,3 \ldots$ do:

1. Uniformly sample $\tau$ blocks of coordinates $S_{k}^{(t)}$.

2. Compute $\alpha_{t} \in(0, \kappa / n]$ using the relation:

$$
\frac{n^{2}}{\kappa^{2}} \alpha_{t}^{2}=\left(1-\alpha_{t}\right) \gamma_{t}+\alpha_{t} \mu_{\kappa},
$$

and set $\gamma_{t+1}=\left(1-\alpha_{t}\right) \gamma_{t}+\alpha_{t} \mu_{\kappa}, \beta_{t}=\frac{\alpha_{t} \mu_{\kappa}}{\gamma_{t+1}}$.

3. Reduce:

$$
y^{(t)}=\frac{1}{\alpha_{t} \gamma_{t}+\gamma_{t+1}}\left(\alpha_{t} \gamma_{t} z^{(t)}+\gamma_{t+1} x^{(t)}\right)
$$

4. for all index $i \in S_{k}$, compute $z_{i}^{(t+1)}$ as

$$
\begin{aligned}
z_{i}^{(t+1)}=\underset{x_{i} \in \mathbb{R}^{N_{i}}}{\operatorname{argmin}} & \frac{n \alpha_{t} L_{i}}{2}\left\|x_{i}-\left(1-\beta_{t}\right) z_{i}^{(t)}-\beta_{t} y_{i}^{(t)}\right\|_{2}^{2} \\
& +\left\langle\nabla_{i} f\left(y^{(t)}\right), x_{i}\right\rangle+\Psi_{i}\left(x_{i}\right),
\end{aligned}
$$

and for all index $i \notin S_{k}$, compute $z_{i}^{(t+1)}$ as

$$
z_{i}^{(t+1)}=\left(1-\beta_{t}\right) z_{i}^{(t)}+\beta_{t} y_{i}^{(t)}
$$

5. Set

$$
x^{(t+1)}=y^{(t)}+\frac{n}{\kappa} \alpha_{t}\left(z^{(t+1)}-z^{(t)}\right)+\frac{\kappa \mu_{\kappa}}{n}\left(z^{(t)}-y^{(t)}\right)
$$

end for

Corollary 1. Suppose that the same conditions in Theorem. 1 hold and further assume that $f$ is $\mu$-strongly convex. In order to obtain $\mathbb{E}\left[F\left(x^{(t)}\right]-F^{*} \leq \epsilon\right.$, it suffices to have the iteration t satisfy

$$
t \geq \frac{n}{\sqrt{\kappa \mu}} \log \frac{C+D \kappa}{\epsilon}
$$

where $C=F\left(x^{(0)}\right)-F^{*}, D=\gamma_{0} R_{0}^{2} / 2$.

The previous single-machine version APCG provided in [Lin et al., 2014] needs $O(n / \sqrt{\mu})$ to achieve $\epsilon$ accuracy, while our DisAPCG further accelerates this rate by a factor $\sqrt{\kappa}$, omitting the $\log$ term. It remains an open problem that whether we can accelerate this rate by $\kappa$.

Remark 1. We have assumed that the coordinates (and their corresponding data) are uniformly distributed in each node. However, the computation power of each node may be different in practice, in which stragglers may slow down the barrier synchronization. To avoid this, nodes can store different amounts of coordinates, depending their computation power, and consequently, each node $k$ has its own mini-batch size $\tau_{k}$. The above theorem still holds as long as each block is sampled with equal probability.

\section{Efficient Implementation}

For the strongly convex case with $\mu>0$, we can choose $\gamma_{0}=\mu / \kappa$ to get a concise version. In this case, we have that $\gamma_{t}=\mu / \kappa$ and consequently, $\alpha_{t}=\beta_{t}=\sqrt{\kappa \mu} / n$. 
As mentioned in Section 2.2, a straightforward implementation of Alg.1 requires full-dimensional operations on $x$ (i.e. $O(N)$ ), which is comparable to a full-gradient step. Lin et al. [2014] provided an equivalent version to their original algorithm, corresponding to the special single-machine case in our paper, with an efficient update step. Here we show that such strategy can also be used in the distributed settings with some modifications. The overall algorithm is summerized in Alg. 2 and the equivalent assertion is made in Proposition 1. Proof can be found in the appendix.

$\overline{\text { Algorithm } 2 \text { DisAPCG without full-dimensional vector op- }}$ erators in the case $\mu_{\kappa}>0$

Input: $x^{(0)} \in \operatorname{dom}(\Psi)$ and convexity parameter $\mu_{\kappa}>0$. Initialize: $\operatorname{set} u^{(0)}=0, v^{(0)}=x^{(0)}, \alpha=\frac{\sqrt{\kappa \mu}}{n}, \rho=\frac{1-\alpha}{1+\alpha}$. Start $K$ nodes with their corresponding data and coordinates. for $t=0,1,2,3 \ldots$ do:

1. Reduce: $\rho^{t+1} u^{(t)}+v^{(t)}$.

2. Uniformly sample $\tau$ blocks of coordinates $S_{k}^{(t)}$.

3. for all $i \in S_{k}^{(t)}$ compute $h_{i}^{(t)}$ as

$$
\begin{gathered}
h_{i}^{(t)}=\underset{h \in \mathbb{R}^{N_{i}}}{\operatorname{argmin}} \frac{n \alpha L_{i}}{2}\|h\|_{2}^{2}+\left\langle\nabla_{i} f\left(\rho^{t+1} u^{(t)}+v^{(t)}\right), h\right\rangle \\
+\Psi_{i}\left(-\rho^{t+1} u_{i}^{(t)}+v_{i}^{(t)}+h\right)
\end{gathered}
$$

4. Let $u^{(t+1)}=u^{(t)}, v^{(t+1)}=v^{(t)}$ and update $i \in S^{(t)}$ as:

$$
u_{i}^{(t+1)}=u_{i}^{(t)}-\frac{1-\frac{n}{\kappa} \alpha}{2 \rho^{t+1}} h_{i}^{(t)}, v_{i}^{(t+1)}=v_{i}^{(t)}+\frac{1+\frac{n}{\kappa} \alpha}{2} h_{i}^{(t)}
$$

Output: $x^{(t)}=\rho^{t} u^{(t)}+v^{(t)}$.

Proposition 1. Algorithm 2 and Algorithm 1 are equivalent with

for all $t \geq 0$.

$$
\begin{aligned}
& x^{(t)}=\rho^{t} u^{(t)}+v^{(t)}, \\
& y^{(t)}=\rho^{t+1} u^{(t)}+v^{(t)}, \\
& z^{(t)}=-\rho^{t} u^{(t)}+v^{(t)},
\end{aligned}
$$

As we can see, the updating step in Alg.2 avoids fulldimensional operations. However, the reduce step $\rho^{t+1} u^{(t)}+$ $v^{(t)}$ still needs $O(N)$ computation cost in general. We can further explore the structure of certain problem to avoid it, as we shall see for the problem of ERM.

\section{Application to Primal-dual ERM Problem}

\subsection{Primal and Dual ERM Problem}

The ERM problem arises ubiquitously in supervised machine learning applications. Let $A_{1}, \ldots, A_{n}$ be vectors in $\mathbb{R}^{d}$, $\phi_{1}, \ldots, \phi_{n}$ be a sequence of convex functions on $\mathbb{R}$, and $g$ be a convex function on $\mathbb{R}^{d}$, the regularized ERM problem is defined as follows:

$$
\underset{w \in \mathbb{R}^{d}}{\operatorname{argmin}}\left\{P(w)=\frac{1}{n} \sum_{i=1}^{n} \phi_{i}\left(A_{i}^{\top} w\right)+\lambda g(w)\right\}
$$

The dual of the above problem is

$$
\underset{x \in \mathbb{R}^{n}}{\operatorname{argmax}}\left\{D(x)=\frac{1}{n} \sum_{i=1}^{n}-\phi_{i}^{*}\left(-x_{i}\right)-\lambda g^{*}\left(\frac{1}{\lambda n} A x\right)\right\},
$$

where $A=\left[A_{1}, \ldots, A_{n}\right]$, and $\phi^{*}, g^{*}$ are conjugate functions of $\phi, g$ respectively.

Notice that in the above problem, the sample size $n$ is the dimensionality when we consider the dual problem. We focus on the strong convexity case, where we need the following assumption that is also standard in the literature of solving the primal and dual ERM problems.

Assumption 3. Each function $\phi_{i}$ is $1 / \gamma$ smooth and the function $g$ is strong convex with parameter 1 .

The above assumption implies that $\phi_{i}^{*}$ is $\gamma$ strong convex and $g^{*}$ is continuous differentiable.

The structure of $D(x)$ matches problem (1) with the equivalent form $F(x)=-D(x)$, where $f(x)=\lambda g^{*}\left(\frac{1}{\lambda n} A x\right)$ and $\Psi(x)=\frac{1}{n} \sum_{i=1}^{n} \phi_{i}^{*}\left(-x_{i}\right)$. In order to match the linear convergence rate assumption, we re-locate the strong convexity of $\phi_{i}^{*}$ and get the final optimization problem as $\operatorname{argmin}_{x \in \mathbb{R}^{n}} F(x)$ where

$$
F(x)=\underbrace{\lambda g^{*}\left(\frac{1}{\lambda n} A x\right)+\frac{\gamma}{2 n}\|x\|_{2}^{2}}_{f(x)}+\underbrace{\frac{1}{n} \sum_{i=1}^{n}\left(\phi^{*}\left(-x_{i}\right)-\frac{\gamma}{2}\left\|x_{i}\right\|_{2}^{2}\right)}_{\Psi(x)} .
$$

We focus on a special case that $g(w)=\frac{1}{2}\|w\|_{2}^{2}$. Such special case implies that we can efficiently compute the partial coordinate gradient and is mostly used as regularization term in ERM problems [Ma et al., 2015]. In this case, we have

$$
\nabla_{i} f\left(y^{(t)}\right)=\frac{1}{\lambda n^{2}} A_{i}^{\top}\left(A y^{(t)}\right)+\frac{\gamma}{n} y_{i}^{(t)}
$$

Besides, we can determine an upper bound for the Lipschitz constant $L_{i} \leq \frac{R^{2}+\lambda \gamma n}{\lambda n^{2}}$, where $R=\max \left\|A_{i}\right\|_{2}, i \in$ $[n]$, and a lower bound for the strong convexity parameter for $f(x)$ with respect to $\|\cdot\|_{L}$ as $\mu \geq \frac{\lambda \gamma n}{R^{2}+\lambda \gamma n}$. Details can be found in the appendix.

\subsection{Numerical Experiments}

We consider minimizing the smoothed hinge loss problem, in order to satisfy the $1 / \gamma$ smooth condition. Precisely, we have

$$
\phi_{i}(a)= \begin{cases}0 & \text { if } a>1 \\ 1-a-\frac{\gamma}{2} & \text { if } a \leq 1-\gamma \\ \frac{1}{2 \gamma}(1-a)^{2} & \text { otherwise }\end{cases}
$$

The conjugate function of $\phi_{i}$ is then as follows:

$$
\phi^{*}(b)= \begin{cases}b+\frac{\gamma}{2} b^{2} & \text { if } b \in[-1,0] \\ \infty & \text { otherwise }\end{cases}
$$

Consequencely, we have

$\Psi_{i}(x)=\frac{1}{n}\left(\phi_{i}^{*}(-x)-\frac{\gamma}{2}\|x\|_{2}^{2}\right)=\left\{\begin{array}{cl}\frac{-x}{n} & \text { if } x \in[0,1] \\ \infty & \text { otherwise }\end{array}\right.$

In the context of primal-dual optimization problem, people often care about the duality gap $P(w(x))-D(x)$, which is 

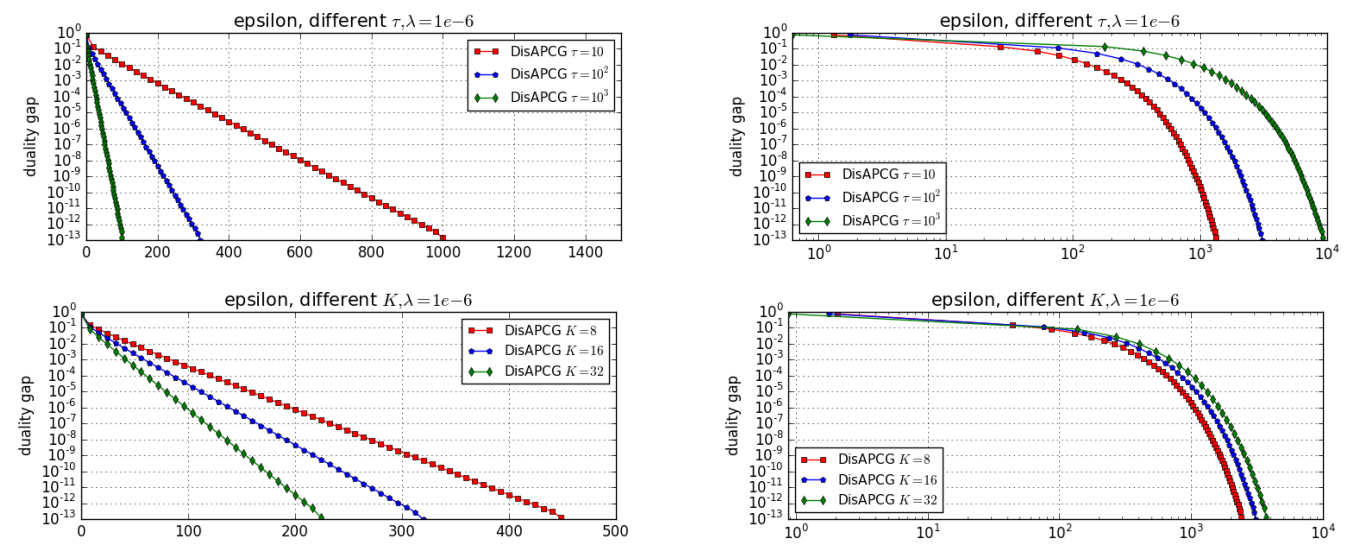

Figure 1: Duality gap vs. the number of iterations, as well as duality gap vs. elapsed time for the Epsilon datasets. We vary the mini-batch size $\tau$ while keep the number of nodes $K$ fixed or vise versa. $\lambda$ is fixed to be $10^{-6}$. The duality gap and elapsed time are shown in log domain while the number of iterations is shown normally to emphasize the linear convergence rate.

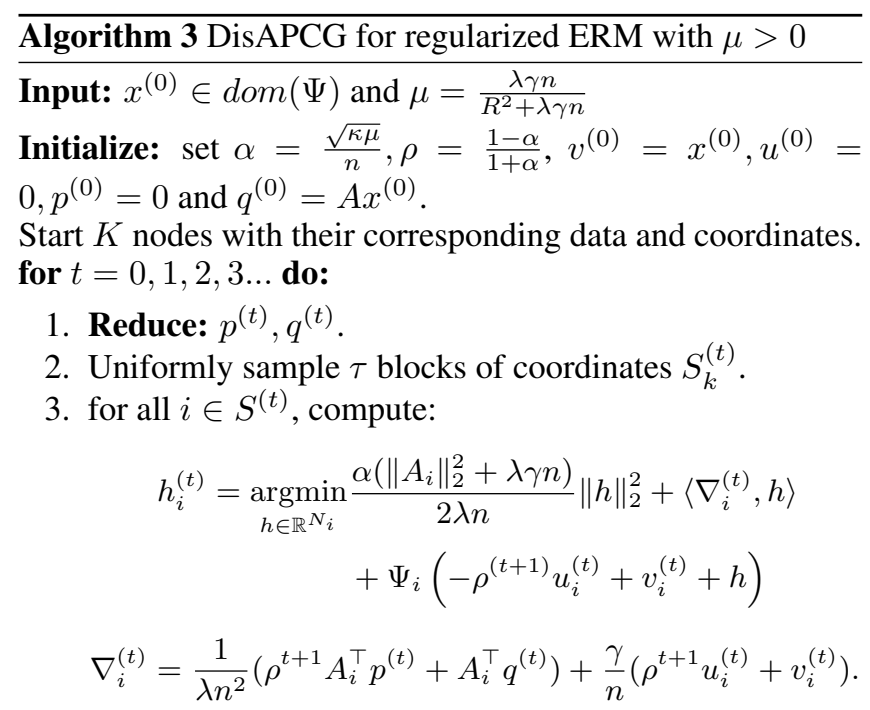

4. Let $u^{(t+1)}=u^{(t)}, v^{(t+1)}=v^{(t)}$ and for all $i \in S^{(t)}$ :

$u_{i}^{(t+1)}=u_{i}^{(t)}-\frac{1-\frac{n}{\kappa} \alpha}{2 \rho^{t+1}} h_{i}^{(t)}, \quad v_{i}^{(t+1)}=v_{i}^{(t)}+\frac{1+\frac{n}{\kappa} \alpha}{2} h_{i}^{(t)}$

Update $p, q$ as

$p^{(t+1)}=p^{(t)}-\frac{1-\frac{n}{\kappa} \alpha}{2 \rho^{t+1}} A_{i} h_{i}^{(t)}, q^{(t+1)}=q^{(t)}-\frac{1+\frac{n}{\kappa} \alpha}{2} A_{i} h_{i}^{(t)}$

Output: primal and dual solutions:

$x^{(t+1)}=\rho^{t+1} u^{(t+1)}+v^{(t+1)}, \quad w^{(t+1)}=\frac{1}{\lambda n}\left(\rho^{t+1} p^{(t+1)}+q^{(t+1)}\right)$

\begin{tabular}{|l|r|r|r|}
\hline datasets & dimension $d$ & sample size $n$ & sparsity \\
\hline epsilon & 2,000 & 100,000 & $100 \%$ \\
covtype & 54 & 581,012 & $22 \%$ \\
RCV1 & 47,236 & 677,399 & $0.16 \%$ \\
\hline
\end{tabular}

Table 1: Information of three binary classification datasets. an upper bound to the gap $D^{*}-D(x)$. An overall discussion about the relation between these gaps can be found in [Dunner et al., 2016]. Here we directly use the duality gap as the statistical indicator.

We implement the algorithms by $\mathrm{C}++$ and openMPI and run them in clusters on Tianhe-II super computer, where in each node we use a single cpu. Experiments are performed on 3 datasets from [Fan and Lin, 2011] whose information is summarized in Table 1.

\section{Influence of mini-batch size $\tau$ and number of nodes $K$}

We first analyze the influence of the mini-batch size $\tau$ and the number of nodes $K$ on the Epsilon dataset. We either vary the mini-batch size $\tau$ on each node with the number of nodes $K$ fixed, or vise versa. Intuitively, a larger mini-batch size $\tau$ means a larger descent in one iteration, however, it needs more computation cost. Similarly, a larger number of nodes $K$ means a larger descent while more communication cost. Therefore, on the one hand, we show the duality gap w.r.t the number of iterations to verify the linear convergence rate and the benefits by increasing computation resources. On the other hand, we show the duality gap w.r.t running time to make clear the trade-off between computation and communication.

The overall results are summarized in Fig.1. In terms of duality gap w.r.t the number of iterations, our DisAPCG algorithm actually achieves a linear convergence rate in all settings, which is consistent with our theory. And the increase of $\tau$ and $K$ does give a large descent in each iteration. Taking a closer look we can see their is a $\sqrt{\kappa}$ accelerating factor in terms of the iteration number, again matching our theoretical findings. For duality gap w.r.t running time, the result suggests that a smaller mini-batch size (e.g., 10) has better performance and more nodes means a faster convergence rate.

\section{Comparison with other solvers}

Now we compare our algorithm with other state-of-art distributed solvers for the primal and dual regularized ERM problem, including the mini-batch version of SDCA in distributed settings [Yang, 2013] (denoted by DisDCA) and Co$\mathrm{CoA}+$ [Ma et al., 2015]. The CoCoA+ solver is an inner-outer 

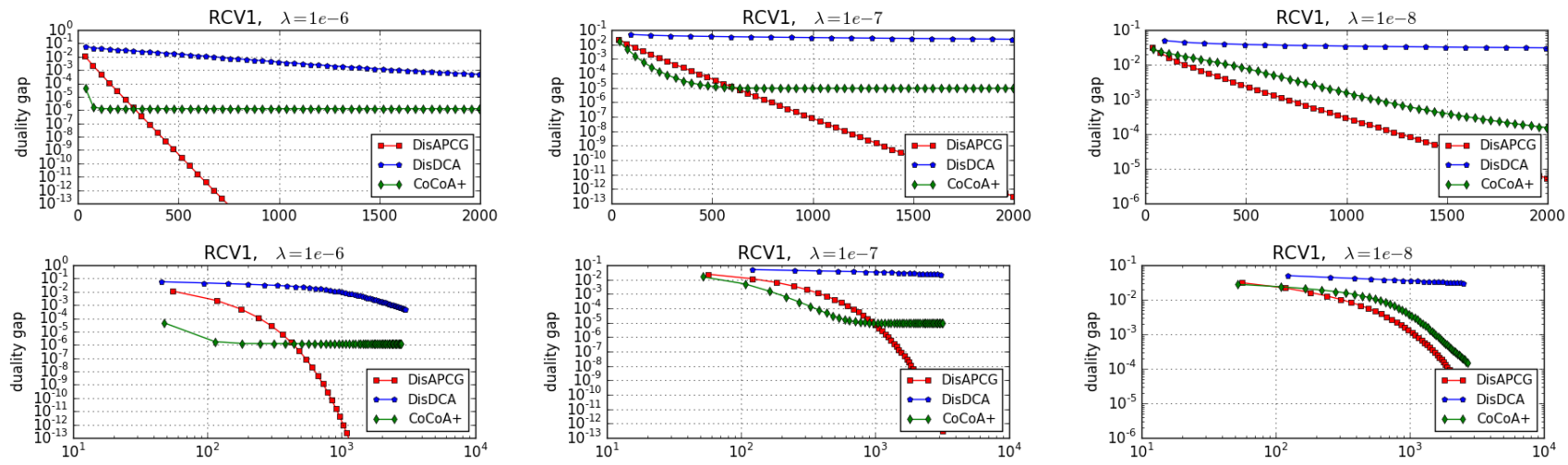

Figure 2: Duality gap vs. the number of iterations, as well as duality gap vs. elapsed time for the RCV1 datasets with number of nodes $K=16, m=H=10^{2}$. $\lambda$ varies from $10^{-6}$ to $10^{-8}$.


Figure 3: Duality gap vs. the number of iterations, as well as duality gap vs. elapsed time for the Covertype datasets with number of nodes $K=16, m=H=10^{2}$. $\lambda$ varies from $10^{-6}$ to $10^{-8}$.

loop scheme that involves a local solver, providing a local approximation to the global problem, and the outer loop is responsible for communication and global parameter updating. Here we follow the original paper [Ma et al., 2015] with SDCA as the local solver. In terms of the number of iterations $H$ for the local SDCA solver, we found that using a relatively small number of iterations (e.g., $10^{2}$ or $10^{3}$ ) achieves the best performance with running time being taken into consideration. Hence we choose $H=10^{2}$ for CoCoAt. For a fair comparison, we set the mini-batch size to be $\tau=10^{2}$ for our DisAPCG method and DisDCA.

We vary $\lambda$ from $10^{-6}$ to $10^{-8}$, which is a relatively hard setting since the strong convexity parameter is small. For all settings, we use $K=16$ nodes. The overall comparison is summarized in Fig.2 and Fig.3. In all settings, the $\mathrm{CoCoA}+$ and our DisAPCG method outperform DisDCA a lot. For the former two, as we can see, when $\lambda$ is relatively large, i.e., $\lambda=10^{-6}$, the $\mathrm{CoCoA}+$ solver reduces the duality gap quickly at the beginning, however, the speed slows down rapidly at an relatively accuracy level, e.g., $10^{-6}$ in the RCV1 dataset. This phenomena happens, no matter with the choice of the number of iterations for the local SDCA solver. In contrast, the DisAPCG algorithm keeps the linear convergence rate all the time and hence achieves better performance in the case that high accuracy is needed. For the most ill condition, i.e., $\lambda=10^{-8}$, our DisAPCG algorithm achieve the best performance, either in terms of iterations or running time, on both datasets. It is worth mentaining that $\mathrm{CoCoA}+$ is a framework that can use any local solver, not only limited in SDCA. As mentioned in Section 2.2, our algorithm can be regarded as a mini-batch version of the APCG algorithm when $K=1$, which supports share-memory level parallel computing in multi-core nodes. A combination of $\mathrm{CoCoA}+$ with our single-machine version of DisAPCG seems to be a good choice to further accelerate the algorithm in practice.

\section{Conclusions}

We have presented a distributed accelerated coordinate methods DisAPCG for the composite convex optimization problem. Our method combines the Nesterov's method and parallel structures, enjoying an accelerated convergence rate for both strongly and non-strongly convex cases. Experiments for the ERM problem show better performance camparing with several other state-of-art solvers, matching our theoretical findings as well.

\section{Acknowledgments}

The work was supported by the National Basic Research Program (973 Program) of China (No. 2013CB329403), NSFC Projects (Nos. 61620106010, 61621136008, 61332007), Tiangong Institute for Intelligent Computing, and the Youth Top-notch Talent Support Program. 


\section{References}

[Bradley et al., 2011] Joseph Bradley, Aapo Kyrola, Danny Bickson, and Carlos Guestrin. Parallel coordinate descent for 11-regularized loss minimization. ICML, 2011.

[Dunner et al., 2016] Celestine Dunner, Simone Forte, Martin Takac, and Martin Jaggi. Primal-dual rates and certificates. ICML, 2016.

[Fan and Lin, 2011] Rong-En Fan and Chih-Jen Lin. Libsvm data: Classification, regression and multi-label. URL: http://www.csie.ntu.edu.tw/ cjlin/libsvmtools/datasets, 2011.

[Fercoq and Richtarik, 2015] Olivier Fercoq and Peter Richtarik. Accelerated, parallel and proximal coordinate descent. SIAM Journal on Optimization, 2015.

[Lin et al., 2014] Qihang Lin, Zhaosong Lu, and Lin Xiao. An accelerated proximal coordinate gradient method and its application to regularized empirical risk minimization. NIPS, 2014.

[Ma et al., 2015] Chenxin Ma, Virginia Smith, Martin Jaggi, Michael Jordan, Peter Richtarik, and Martin Takac. Adding vs. averaging in distributed primal-dual optimization. ICML, 2015.

[Necoara and Clipici, 2013] Ion Necoara and Dragos Clipici. Efficient parallel coordinate descent algorithm for convex optimization problems with separable constraints: application to distributed mpc. Journal of Process Control, 2013.

[Nesterov, 1983] Yurii Nesterov. A method of solving a convex programming problem with convergence rate $\mathrm{o}(1 / \mathrm{k} \hat{2})$. Soviet Mathematics Doklady, 1983.

[Nesterov, 2012] Yurii Nesterov. Efficiency of coordinate descent methods on huge-scale optimization problems. SIAM Journal on Optimization, 2012.

[Richtarik and Takac, 2013a] Peter Richtarik and Martin Takac. Distributed coordinate descent method for learning with big data. arXiv:1310.2059, 2013.

[Richtarik and Takac, 2013b] Peter Richtarik and Martin Takac. Parallel coordinate descent methods for big data optimization. arXiv:1212.0873v2, 2013.

[Shalev-Shwartz and Zhang, 2014] Shai Shalev-Shwartz and Tong Zhang. Accelerated proximal stochastic dual cooridinate ascent for regularized loss minimization. ICML, 2014.

[Takac and Richtarik, 2015] Martin Takac and Peter Richtarik. Distributed mini-batch sdca. arXiv:1507.08322v1, 2015.

[Wright, 2015] Stephen Wright. Coordinate descent algorithms. Mathematical Programming 151.1, 3-34., 2015.

[Xiao and Lu, 2013] Lin Xiao and Zhaosong Lu. On the complexity analysis of randomized block-coordinate descent methods. arXiv:1304.4723, 2013.

[Yang, 2013] Tianbao Yang. Trading computation for communication: Distributed stochastic dual coordinate ascent. NIPS, 2013. 